\title{
Dor lombar crônica e dor nos membros inferiores em idosas: etiologia em revisão
}

\author{
Low back pain and pain in the lower extremity in aged: etiology in review
}

\author{
Renata Maraschin ${ }^{[\mathrm{a}]}$, Péricles Saremba Vieira ${ }^{[\mathrm{b}]}$, Camila Pereira Leguisamo $^{[\mathrm{c}]}$, \\ Fracieli Dal'Vesco $^{[\mathrm{d}]}$, Juceléia Pertile Santi ${ }^{\mathrm{e}]}$
}

[a] Fisioterapeuta, Mestranda em Envelhecimento Humano pela Universidade de Passo Fundo (UPF), Passo Fundo, RS - Brasil, e-mail: rechinpf@gmail.com

[b] Professor Doutor do Programa de Pós-graduação em Envelhecimento Humano da Universidade de Passo Fundo (UPF), Passo Fundo, RS - Brasil, e-mail: psvieira@upf.br

[c] Professora Mestre do curso de Fisioterapia da Universidade de Passo Fundo (UPF), Passo Fundo, RS - Brasil, e-mail: camila@upf.br

[d] Fisioterapeuta, Mestranda em Envelhecimento Humano pela Universidade de Passo Fundo (UPF), Passo Fundo, RS - Brasil, e-mail: francieli.dalvesco@gmail.com

[e] Educadora Física, Mestranda em Envelhecimento Humano pela Universidade de Passo Fundo (UPF), Passo Fundo, RS - Brasil, e-mail: juceper@gmail.com

\section{Resumo}

Introdução: A relação entre dor lombar crônica, dor nos membros inferiores e dependência funcional em idosos evidencia-se pelo comportamento da dor durante a execução de atividades da vida diária. Permanecer em pé, puxar ou empurrar objetos grandes e caminhar podem desencadear dor e causar grande sofrimento para os idosos. Objetivo: Identificar as causas descritas na literatura relativas à dor lombar crônica e à dor nos membros inferiores em idosas. Método: Realizou-se busca, nas bases de dados Medline e Pubmed, de literatura em inglês publicada entre 1992 e 2008. Resultados: Dos 48 artigos encontrados, 33 foram analisados. Entre as causas não mecânicas de dor, foram mencionadas fraturas vertebrais por compressão relacionadas à osteoporose, estenose espinhal lombar, metástases, malignidade, síndrome da cauda equina, bacteremia, infecções geniturinárias, tuberculose, polimialgia reumática, aneurisma aórtico, Doença de Paget e Doença de Parkinson. Entre as causas mecânicas, prevalentes em idosos e relacionadas ao sistema musculoesquelético, foram encontradas indefinições quanto à relação de causa e efeito estabelecida entre osteoartrite e dor. Em mulheres, a relação entre menopausa, uso de estrogênio e ocorrência de osteoporose-fratura-dor parece ainda pouca esclarecida. Associação entre dor lombar e dor nos membros inferiores pode sugerir causas mecânicas ou outras causas, visto que osteoartrite e distúrbios circulatórios, comuns em idosas, nem sempre são fontes de dor. Conclusão: 
Pela análise da literatura concluiu-se que, além da etiologia mencionada, outras possibilidades de causas, ainda não completamente identificadas e compreendidas, podem estar na origem da dor lombar e da dor nos membros inferiores de idosas.

Palavras-chave: Etiologia. Dor lombar. Idosos. Membros inferiores.

\begin{abstract}
Introduction: Relation between low back pain, pain in lower extremity and functional dependence in aged is proven for the behavior of pain during the execution of activities of daily life. To remain in foot, to pull or to push great objects and to walk can unchain pain and cause great suffering for the aged ones. Objective: Ahead of this, the present study had as objective to identify causes in literature relative to chronic lumbar pain and pain in lower extremity in aged. Method: Search in the databases Medline and Pubmed of literature in English published between 1992 and 2008 was fullfilled. Results: Of 48 found articles, 33 had been analyzed. Not-mechanical causes of pain had been mentioned as vertebral compression fractures related to osteoporosis, lumbar spinal stenosis, metastatic cancer, malignancy, cauda equina syndrome, bacteremia, genitourinary infections, tuberculosis, polymyalgia rheumatica, aortic aneurysm, Paget disease and Parkinson disease. Between the mechanical causes, prevalent in aged and related to the musculoskeletal system, had been found doubts about relation of cause and effect established between osteoartritis and pain. In women, relation between menopause, estrogen use and occurrence of osteoporosisfracture-pain still seems little clarified. Association between lumbar pain and pain in lower extremity can suggest mechanical causes or other causes, because circulatory disease and osteoartritis, common in aged, not even are pain sources. Conclusion: For the analysis of literature we concluded that beyond the mentioned etiology, other possibilities of causes, not yet completely identified and understood, can be in the origin of lumbar pain and pain in lower extremity of aged.
\end{abstract}

Keywords: Etiology. Lumbar pain. Aged. Lower extremity.

\title{
Introdução
}

A proporção de idosos (com 60 anos ou mais), em quase todos os países desenvolvidos e em desenvolvimento do mundo, está aumentando mais rápido do que qualquer outro grupo etário (1). Dados de 2007 da Organização Mundial de Saúde (OMS) mostram que a população mundial idosa atual é de 650 milhões de pessoas e que, em 2050, é provável que esse número alcance 2 bilhões (2). No Brasil, segundo dados da Pesquisa Nacional por Amostra de Domicílios (Pnad), em 2007 já havia 20 milhões de idosos, correspondendo a 10,5\% da população (3). Além desse aumento, ainda em 1988 (4) já era possível observar tendência de feminilização da população idosa, pois as mulheres geralmente vivem por mais tempo e se casam com homens mais velhos. Dados do IBGE de 2000 confirmam essa tendência (5).

Nesse contexto, as enfermidades crônicas associadas ao envelhecimento surgem como preocupação, sobretudo, relacionadas à qualidade de vida neste período. Isso porque tais enfermidades apresentam relação estatisticamente significante com o nível de dependência funcional dos idosos (6). Essas enfermidades podem estar relacionadas a relatos de dor, os quais, por sua vez, podem relacionar-se à incapacidade funcional. A relação entre dor e dependência funcional evidencia-se pelo comportamento da dor durante a execução de atividades da vida diária por idosos. Assim, observa-se relação entre dor lombar em idosas e dificuldade para permanecer em pé em um lugar, para puxar ou empurrar objetos grandes e para caminhar (7).

Também é possível identificar relação entre dor lombar e dor nos membros inferiores, sintoma das enfermidades crônicas relacionadas ao sistema locomotor, como condição de alta prevalência na população feminina idosa $(8,9)$. A dor lombar foi associada, por mulheres idosas, à dor nos membros inferiores e à dificuldade para realizar tarefas cotidianas (7). Ao mesmo tempo, os fatores psicológicos não explicam a relação 
entre dor lombar e nos membros inferiores e incapacidade funcional em mulheres idosas (10). A ocorrência de dor lombar e de relatos de dor nos membros inferiores e sua decorrente incapacidade funcional para idosas parecem ser entendidos, na literatura, menos como condição psicológica e mais como condição biológica. Esse tende a ser assunto de interesse do fisioterapeuta, como profissional que se ocupa das questões relacionadas ao movimento humano e, por meio de sua atividade profissional, possibilita melhor qualidade de vida aos indivíduos. Diante disso é possível questionar: como o fisioterapeuta vem realizando suas atividades profissionais no tratamento de dor lombar e de dor nos membros inferiores de idosas? Que referenciais etiológicos são utilizados na aplicação de técnicas fisioterápicas que visem a aliviar/suprimir a dor lombar e nos membros inferiores de idosas? Como a literatura descreve tais referenciais? Apenas como doenças isoladas da coluna lombar e dos membros inferiores ou também como condições decorrentes de compensações ocasionadas por disfunções em outros segmentos do corpo?

$\mathrm{Na}$ tentativa de buscar respostas a estas questões, o presente artigo teve como objetivo identificar as causas, descritas na literatura, de dor lombar e de dor nos membros inferiores em idosas, e, a partir disso, disponibilizar subsídios para o exercício profissional pautado, cada vez mais, no conhecimento e nas particularidades do envelhecimento humano.

\section{Materiais e métodos}

Realizou-se busca, nas bases de dados Medline e Pubmed, de artigos em inglês publicados entre 1992 e 2008 relacionados à dor lombar e à dor na extremidade inferior em idosos. Buscaram-se qualificadores em inglês para os termos etiologia, dor lombar, idosos e membros inferiores. Os qualificadores encontrados e utilizados em combinação na estratégia de busca dos artigos foram, respectivamente: etiology, low back pain, aged, lower extremity. Após realizada a busca, artigos que fizessem referência à dor lombar e à dor nos membros inferiores somente em crianças e adultos jovens foram excluídos. Foram incluídos artigos que fizessem referência às possíveis causas de dor lombar e dor nos membros inferiores em idosos (independente do sexo) e que sugerissem condições possivelmente envolvidas na etiologia da dor. Assim, dos 48 artigos encontrados, 33 foram analisados. Quatro referências em português foram mencionadas na introdução, dada a importância de seu conteúdo, e a fim de contextualizarem o problema proposto pela pesquisa.

\section{Resultados}

Quando comparada com outros grupos etários, a dor lombar crônica prevalente em idosos (11-14) é considerada de difícil diagnóstico (15), pois há maior número de causas possíveis (16) e diferenças quanto à natureza da causa (17), o que pode ser observado no Quadro 1. Nessa população, a dor pode ser resultado de causas mecânicas (dor aumenta com movimento ou carga física) ou causas não mecânicas (dor presente no repouso) (18) e somente em 15\% dos casos de dor lombar crônica é possível determinar a etiologia da dor (13).

Quadro 1 - Principais achados na literatura entre 1992 e 2008 sobre a etiologia da dor lombar e dor nos membros inferiores em idosas

(Continua)

\section{Principais achados}

Dor musculoesquelética, com frequência incorretamente atribuída à artrite, é a maior causa de inabilidade em idosos. Fibromialgia, dor miofascial, progressão e acumulação de problemas dolorosos segmentares podem determinar explicações não articulares para a dor
Autores

Leveille SG et al., 2007 (10) 
Quadro 1 - Principais achados na literatura entre 1992 e 2008 sobre a etiologia da dor lombar e dor nos membros inferiores em idosas

\section{Principais achados}

Dor lombar que irradia no quadril, nádega ou perna é relativamente comum em mulheres caucasianas no período pós-menopausa. Em 9\%, os sintomas na perna e na lombar foram aliviados ao sentar, o que pode sugerir causas mecânicas

- Relação entre disco, articulações facetárias e alterações degenerativas como fonte de dor crônica na coluna lombar em idosos

- Alterações da coluna lombar ocasionadas pelo avanço da idade e detectadas radiologicamente são fracamente relacionadas à presença de dor e encontradas em pacientes sintomáticos e assintomáticos

Em 15\% dos casos de dor lombar crônica é possível determinar a etiologia da dor

A maioria dos pacientes idosos apresenta etiologia da dor lombar descrita como desconhecida, causa mecânica não específica, dor lombar regional ou inespecífica. Pouco investigada

- Fratura vertebral por compressão relacionada à osteoporose

- Estenose espinhal lombar

- Metástases

- Síndrome da Cauda Equina

- Doenças infecciosas como bacteremia, infecções geniturinárias e tuberculose

- Dor usualmente associada à atividade, evento inicial específico causador nem sempre identificado

- Entorses de ligamentos ou de músculos posteriores, componente radicular e espasmo muscular

- Causas mecânicas não específicas

- Polimialgia Reumática

- Aneurisma Aórtico

- Doença de Paget

- Doença de Parkinson

- Osteoporose com fratura vertebral por compressão

- Pacientes idosos tendem a mostrar marcada discrepância entre achados radiológicos de degeneração e dor efetiva

- Segmentos instáveis e espondiloartrose podem levar a estiramento muscular sempre que estabilidade secundária insuficiente e perda do equilíbrio muscular estão envolvidas no surgimento da dor. Isquialgia causada pela protrusão de um disco intervertebral ou uma síndrome lombo vertebral causada por instabilidade muscular são achados muito menos frequentemente como causa de dor lombar em idosos

- Desordens mecânicas mais relacionadas à doença degenerativa discal e articular - Alterações da coluna lombar ocasionadas pelo avanço da idade e detectadas radiologicamente são fracamente relacionadas à presença de dor e são encontradas em pacientes sintomáticos e assintomáticos

- Risco de dor é encontrado com o aumento do número de fraturas vertebrais por compressão

\section{Autores}

Vogt MT et al., 2002 (11)

Manchikanti L et al., 2008 (12)

Manchikanti L et al., 2004 (13)

Cecchi F et al., 2006 (14)

Lazaro L 4 TH, Quinet RJ, 1994 (15)

Mazanec DJ, 1999 (16)

Baumgartner H, 1996 (17)

Wing PC, 2001 (18) 
Quadro 1 - Principais achados na literatura entre 1992 e 2008 sobre a etiologia da dor lombar e dor nos membros inferiores em idosas

\section{Principais achados}

Malignidade

Doença de Parkinson

Maioria dos pacientes idosos apresenta etiologia da dor lombar descrita como desconhecida, causa mecânica não específica, dor lombar regional ou inespecífica

Em espécimes de coluna lombar maiores que 60 anos, o mais comum nível de alterações artríticas parece ser L4-L5. Homens têm maior prevalência e grau de artrose facetária do que mulheres

Anormalidades do disco intervertebral, descritas em exames de imagem em indivíduos assintomáticos, são associadas com, mas não sinônimos para osteoartrite

Osteofitose facetária aparece precocemente em processos degenerativos, precedendo a osteofitose da borda vertebral, característica da degeneração do disco intervertebral. Degeneração facetária predomina em indivíduos jovens. Degeneração óssea em ambas as localizações (disco e facetas) igualmente reflete a progressão da degeneração nos tecidos moles

Mulheres geralmente referem mais sintomas dolorosos do que os homens

- Os locais mais comuns de dor no membro inferior, em ambos os sexos, são quadris e joelhos

- Metade de homens e mulheres com dor generalizada, com mínima a moderada dor em um ou mais locais não possuem radiografia com osteoatrite de joelhos. Outras condições patológicas sublinham o problema em muitos idosos

- Risco de dor é encontrado com o aumento do número de fraturas vertebrais por compressão

- Risco de uma fratura vertebral, a partir dos 50 anos, é de $16 \%$ em mulheres brancas e apenas $5 \%$ em homens brancos

Baixo índice de massa corporal, fumo, baixo consumo de leite durante a gravidez, baixos níveis de atividade física diária, queda e consumo regular de antiácidos contendo alumínio, independentemente, aumentam o risco de uma primeira fratura vertebral. Mulheres usando estrogênio e aquelas que participam de atividade física recreacional têm baixo risco

Risco de dor é encontrado com o aumento do número de fraturas vertebrais por compressão

Risco de dor é encontrado com o aumento do número de fraturas vertebrais por compressão

- Taxa de fratura vertebral apresenta-se elevada em homens e mulheres

- Entre 50 e 79 anos, $12 \%$ a 20\% das mulheres apresentam uma ou mais deformidades vertebrais, que representam significativo maior risco de fraturas vertebrais subsequentes (aproximadamente cinco vezes)

\section{Autores}

Henschke N, Maher CG, Refshauge KM, 2007 (19)

Burton RR, 2008 (20)

Ponka D, Kirlew M, 2007 (21)

Eubanks JD et al., 2007 (22)

Borenstein D, 2004 (23)

Eubanks JD et al., 2007 (24)

Astin M, Lawton C, Hirst M, 1996 (25)

Leveille SG et al., 2005 (26)

Melton L J, 1997 (27)

Nevitt MC et al., 2005 (28)

Hartvigsen J et al., 2004 (29)

Nevitt MC et al., 1998 (30)

Krieg MA, Lamy O, 2004 (31) 
Quadro 1 - Principais achados na literatura entre 1992 e 2008 sobre a etiologia da dor lombar e dor nos membros inferiores em idosas

(Conclusão)

\section{Principais achados}

Uso de estrogênio pós-menopausa é associado com um aumento da probabilidade de dor lombar e prejuízo da função lombar em mulheres brancas idosas

Deformidades vertebrais em homens são, pelo menos, comuns, se não mais prevalentes, do que em mulheres

Embora seja possível observar aumentado risco de fraturas relacionado às deformidades vertebrais como uma possível causa de dor lombar, sintomas lombares foram associados com limitações em atividades funcionais mesmo na ausência de deformidade vertebral em mulheres idosas

Deformidades vertebrais em mulheres idosas causam dor substancial, inabilidade ou perda de altura somente se a proporção de perda vertebral cai quatro desvios padrões abaixo do valor normal. Dor lombar intensa não pode ser atribuída a deformidades vertebrais, sugerindo outras causas

Problemas lombares em mulheres idosas são associados com doença cardiovascular autorrelatada, mas não com objetivamente avaliada doença arterial da extremidade inferior

- Dor osteoarticular da extremidade inferior foi significativamente associada com idosas de menor idade, raça negra, baixa renda, inabilidade mais severa, mais alto nível de depressão, baixo uso do álcool, índice de massa corporal elevado, creatinina elevada, uso dos nitratos, osteoartrite das mãos, dos quadris, ou dos joelhos e doença do disco ou estenose espinal

- Os locais mais comuns de dor no membro inferior, em ambos os sexos, são quadris e joelhos

Artrite patelo-femoral é causa de inabilidade em idosas

- Em amostra de italianos acima de 65 anos, a prevalência de dor no joelho foi quase o dobro de dor no quadril. Enquanto ambas as condições foram relacionadas à dor em outras articulações e específico prejuízo articular (dor lombar, abdução de quadril reduzida, redução da força muscular e aumento da flexibilidade do tronco), apenas dor no quadril foi relacionada à pobre autoavaliação de saúde e somente dor no joelho à sobrecarga mecânica

- Dor no joelho foi relacionada a alto índice de massa corporal, à dor no quadril e à dor no pé

- Dor nos pés e inabilidade é mais provável em mulheres (56\%) que em homens $(24 \%)$, com maior prevalência entre elas de deficiência na extremidade inferior e de relatos de dor em vários outros locais (peito, mãos, coluna lombar, quadril, joelhos e panturrilha), indicando que dor severa no pé, com frequência, ocorre como parte de um problema doloroso mais generalizado

- Causas de dor nesses locais são essencialmente relacionadas com distúrbios

osteomusculares (condições artríticas) e circulatórios (edema bilateral de tornozelo).
Autores

Musgrave DS et al., 2001 (32)

Jones G et al., 1996 (33)

Edmond SL et al., 2005 (34)

Ettinger B et al., 1992 (35)

Vogt M'T, Nevitt MC, Cauley JA, 1997 (36)

Pahor M et al., 1999 (37)

Creighton D et al., 2007 (38)

Cecchi F et al., 2008 (39)

Leveille SG et al., 1998 (40)

Fisioter Mov. 2010 out/dez;23(4):627-39 
$\mathrm{Na}$ idade avançada, a dor lombar é, com menor frequência, causada por estresse ocupacional e fatores psicossomáticos (17) e as causas prováveis descritas na literatura são: fratura vertebral por compressão relacionada à osteoporose (15-17), estenose espinhal lombar (15-18), metástases (15), malignidade (19), síndrome da cauda equina (15) e doenças infecciosas como bacteremia, infecções geniturinárias e tuberculose (15), polimialgia reumática (16), aneurisma aórtico (16), Doença de Paget (16) e Doença de Parkinson (16, 20).

Quando nenhuma dessas causas está presente, a etiologia da dor lombar é descrita como desconhecida, causa mecânica não específica, dor lombar regional ou inespecífica. Apresentada pela maioria dos pacientes idosos $(14-16,21)$, essa etiologia, com frequência, compreende condições que são potencialmente reversíveis, que podem ser alvo de intervenções específicas, mas que ainda são pouco investigadas em idosos (14). A dor é usualmente associada à atividade, embora um evento inicial específico causador nem sempre seja identificado (15). Todavia, desordens mecânicas são verdadeiramente musculoesqueléticas e em idosos, embora possam incluir entorses de ligamentos ou de músculos posteriores, componente radicular e espasmo muscular (15), estão mais relacionadas à doença degenerativa discal e articular (18).

\section{Degeneração, disco e articulações facetárias como fontes de dor}

Parece razoável admitir a relação entre disco, articulações facetárias e alterações degenerativas como fonte de dor crônica na coluna lombar em idosos $(12,13,22,23)$. Isso porque, embora o pico de idade do início dos sintomas lombares seja a meia idade, problemas estruturais aumentam com a idade. Alterações degenerativas que são visíveis em radiografias são quase universais em pessoas acima dos 80 anos de idade, com pequena porcentagem apresentando discos que aparecem normais nas radiografias (18). $O$ esqueleto axial demonstra as mesmas alterações clássicas de perda de cartilagem, instabilidade articular e osteofitose, características de doença sintomática nos membros (23). Corroborando com essa informação, estudo (22) anatômico e epidemiológico de artrose facetária em colunas lombares cadavéricas mostrou que este achado esteve presente em 100\% naqueles espécimes maiores que 60 anos, que o mais comum nível de alterações artríticas parece ser L4-L5 e que homens têm maior prevalência e grau de artrose facetária do que mulheres.

Entretanto, a localização da dor causada pela degeneração facetária torna-se difícil, pois essas estruturas apresentam entrada sensorial de dois níveis espinhais (23). Também, pacientes idosos tendem a mostrar uma marcada discrepância entre achados radiológicos de degeneração e dor efetiva (17). Isso porque as alterações da coluna lombar ocasionadas pelo avanço da idade e detectadas radiologicamente são fracamente relacionadas à presença de dor e são encontradas em pacientes sintomáticos e assintomáticos $(18,12,23)$. Ainda, anormalidades do disco intervertebral, descritas em exames de imagem em indivíduos assintomáticos, são associadas com, mas não sinônimos para osteoartrite (23).

Se for admitida a relação entre disco, articulações facetárias e alterações degenerativas como fonte de dor crônica na coluna lombar em idosos $(12,13,22,23)$, então parece razoável perguntar: são as alterações degenerativas do disco que determinam alterações degenerativas nas articulações facetárias ou o inverso? Ou ainda uma terceira possibilidade: é possível que as alterações degenerativas do disco e das articulações facetárias ocorram simultaneamente? Pelo exame de 647 colunas lombares de esqueletos, estudo (24) sugere que osteofitose facetária aparece precocemente em processos degenerativos, precedendo a osteofitose da borda vertebral, característica da degeneração do disco intervertebral. Quando as facetas começam a se deteriorar com a idade, a osteofitose da borda vertebral ultrapassa a osteofitose facetária. Estes dados desafiam a crença de que a degeneração facetária segue a degeneração discal; antes, parece que esta progride mais rapidamente em anos mais tarde, mas a degeneração facetária predomina em indivíduos jovens. Há evidências (24) de que a degeneração óssea em ambas as localizações (disco e facetas) igualmente refletem na progressão da degeneração nos tecidos moles. Segmentos instáveis e espondiloartrose podem levar a estiramento muscular sempre que estabilidade secundária insuficiente e perda do equilíbrio muscular estiverem envolvidas no surgimento da dor (17). Entretanto, isquialgia ocasionada por protrusão de um disco intervertebral ou uma síndrome lombo vertebral causada por instabilidade muscular são achados muito menos frequentemente como causa de dor lombar em idosos (17). 
As alterações degenerativas da coluna lombar, seus efeitos sobre a musculatura e sua relação com a dor em idosos parecem também sofrer influência do gênero. A que se deve o fato de homens apresentarem grau maior de artrose facetária do que mulheres (22)? Que fatores relacionados ao gênero podem estar envolvidos? É possível que outras causas de dor lombar estejam presentes apenas em mulheres idosas? Quais seriam esses fatores? Podem esses fatores estabelecer relação etiológica com a dor nos membros inferiores em idosas?

\section{Dor lombar e dor nos membros inferiores em idosos: algumas diferenças e semelhanças entre os sexos e principais causas de dor em mulheres}

As observadas diferenças entre homens e mulheres na prevalência e consequências da dor lombar crônica permanecem pouco esclarecidas. Embora pesquisas indiquem que os fatores de percepção da dor, como flutuações do ciclo menstrual na sensibilidade da dor e o fato de que a mulher geralmente refere mais sintomas dolorosos do que o homem (25), podem ajudar a explicar as diferenças na dor relatada por adultos jovens, pouca pesquisa tem examinado a diferença entre idosos (26). No que se refere à literatura analisada sobre diferenças entre homens e mulheres idosos na prevalência e consequências da dor lombar crônica, foram identificadas indefinições e discrepâncias que apontam em algumas direções:

1) entre mulheres idosas há relato de maior número de regiões dolorosas e maior prevalência de dor generalizada (dor lombar e dor em membros superiores e inferiores bilateralmente) relacionada a fatores como alto índice de massa corporal, pressão sanguínea sistólica alta e sintomas depressivos. Em homens e mulheres, a dor foi associada com média ou pobre autoavaliação de saúde, história de dor lombar antes dos 65 anos de idade e inabilidade (26);

2) embora o pico de dor lombar seja em torno dos 40 anos, foi encontrado um segundo pico de incidência de dor lombar em mulheres idosas. Isso pode estar relacionado aos efeitos da osteoporose, o que não é um fator de risco por si, embora aumento no risco de dor seja encontrado com o aumento do número de fraturas vertebrais por compressão (18, 27-30). A taxa de fratura vertebral apresenta-se elevada em homens e mulheres $(27,31)$, mas como as mulheres estão vivendo mais, o risco de uma fratura vertebral a partir dos 50 anos é de 16\% em mulheres brancas e de apenas 5\% em homens brancos $(27,28)$. Fratura não vertebral prévia, baixa densidade mineral óssea em todos os locais, e especificamente na coluna lombar (27), baixo índice de massa corporal, fumo, baixo consumo de leite durante a gravidez, baixos níveis de atividade física diária, queda e consumo regular de antiácidos contendo alumínio, independentemente, aumentam o risco de uma primeira fratura vertebral. Mulheres usando estrogênio e aquelas que participam de atividade física recreacional têm um baixo risco (28). Todavia, outros dados (32) indicam que o uso de estrogênio pós-menopausa é associado com um aumento da probabilidade de dor lombar e prejuízo da função lombar em mulheres brancas idosas;

3) deformidades vertebrais em homens são, pelo menos, comuns, se não mais prevalentes, que em mulheres (33). Entre 50 e 79 anos, $12 \%$ a $20 \%$ das mulheres apresentam uma ou mais deformidades vertebrais prevalentes. Manifestação mais comum de osteoporose, as deformidades representam significativo maior risco de fraturas vertebrais subsequentes (aproximadamente cinco vezes) (31). Isso porque a densidade mineral óssea foi mais fortemente associada com deformidades bicôncavas do que deformidades em cunha ou em esmagamento, apresentando maior força de associação com prevalência de fraturas vertebrais. Já a osteofitose espinhal falsamente eleva a densidade mineral óssea, sem um concomitante decréscimo no risco de fraturas (33). Embora seja possível observar aumentado risco de fraturas relacionado às deformidades vertebrais como uma possível causa de dor lombar, sintomas lombares foram associados com limitações em atividades funcionais mesmo na ausência de deformidade vertebral em mulheres idosas (34). Talvez porque deformidades vertebrais em mulheres idosas causem dor substancial, inabilidade ou perda de altura somente se a proporção de perda vertebral cai quatro desvios padrões abaixo do valor normal. Assim, dor lombar intensa não pode ser atribuída a deformidades vertebrais, 
sugerindo outras causas (35) como, por exemplo, distúrbios do sistema cardiovascular. Resultados indicam que problemas lombares em mulheres idosas são associados com doença cardiovascular autorrelatada, mas não com objetivamente avaliada doença arterial da extremidade inferior (36);

4) dor lombar que irradia no quadril, nádega ou perna é relativamente comum em mulheres caucasianas no período pós-menopausa. Em 9\%, os sintomas na perna e na lombar foram aliviados ao sentar, o que pode sugerir causas mecânicas (11);

5) a dor osteoarticular da extremidade inferior foi significativamente associada com idosas de menor idade, raça negra, baixa renda, inabilidade mais severa, mais alto nível de depressão, baixo uso do álcool, índice de massa corporal elevado, creatinina elevada, uso dos nitratos, osteoartrite das mãos, dos quadris, ou dos joelhos e doença do disco ou estenose espinal (37). Os locais mais comuns de dor no membro inferior, em ambos os sexos, são quadris e joelhos $(26,37)$;

6) a artrite patelo-femoral é causa de inabilidade em idosas (38). Entretanto, aproximadamente metade de homens e mulheres com dor generalizada - definida como dor na extremidade superior (mão e punho), extremidade inferior (quadril, joelho ou pé) e esqueleto axial (costas ou peito) - e com mínima a moderada dor em um ou mais locais não possui radiografia com osteoatrite de joelhos $(10,26)$, sugerindo que outras condições patológicas sublinham o problema em muitos idosos (26). Em anos recentes, tem se tornado mais evidente que a dor musculoesquelética, com frequência incorretamente atribuída à artrite, é a maior causa de inabilidade em idosos (10);

7) informações clínicas podem determinar explicações não articulares para a dor, como fibromialgia, dor miofascial e progressão e acumulação de problemas dolorosos segmentares (10). Em uma amostra de italianos acima de 65 anos, a prevalência de dor no joelho foi quase o dobro da dor no quadril. Enquanto ambas as condições foram relacionadas à dor em outras articulações e específico prejuízo articular (dor lombar, abdução de quadril reduzida, redução da força muscular e aumento da flexibilidade do tronco), apenas dor no quadril foi relacionada à pobre autoavaliação de saúde e somente dor no joelho à sobrecarga mecânica (39). Isso pode estar relacionado à associação de dor na extremidade inferior em idosas e aumento da probabilidade de dificuldade para subir escadas, mas não dificuldade para caminhar (10, 37);

8) dor no joelho foi relacionada a alto índice de massa corporal, à dor no quadril e à dor no pé $(37,39)$. Mulheres idosas estão em risco particular para dor no pé se comparadas aos homens, por causa de sua maior prevalência de deformidades nos pés, bem como osteoartrite e artrite reumatoide. Dor nos pés e inabilidade são mais prováveis em mulheres (56\%) do que em homens (24\%), com maior prevalência entre elas de deficiência na extremidade inferior e de relatos de dor em vários outros locais (peito, mãos, coluna lombar, quadril, joelhos e panturrilha), indicando que dor severa no pé, com frequência, ocorre como parte de um problema doloroso mais generalizado. Causas de dor nesses locais são essencialmente relacionadas com distúrbios osteomusculares (condições artríticas) e circulatórios (edema bilateral de tornozelo). Ainda, o aumento na severidade de dor no pé foi associado com pior desempenho nos testes funcionais para membros inferiores, tais como caminhar, independentemente da prevalência de doenças crônicas ou outra dor na extremidade inferior. Entre as mulheres que tiveram sintomas definidos de osteoatrite de mão e joelho, a porcentagem de mulheres com dor crônica nos pés foi duas vezes maior que a observada em mulheres sem estas condições. Modestas diferenças foram observadas na porcentagem de mulheres que tiveram dor no pé com presença de insuficiência cardíaca congestiva e diabetes, mas as diferenças não foram estatisticamente significantes (40).

\section{Considerações finais}

Quando se trata de mencionar a etiologia não mecânica da dor lombar em idosos, a literatura aponta causas definidas (tumores, fraturas, infecções). A mesma clareza, contudo, parece não estar evidente no que se refere à etiologia mecânica da dor. Tal fato causa surpresa, visto que essa etiologia é prevalente em idosos e 
frequentemente atribuída a distúrbios musculoesqueléticos, especialmente osteoartrite, achado comum nessa população. Restam, portanto, indefinições e discrepâncias sobre os determinantes da relação osteoartrite-dor e da influência etiológica do gênero na dor lombar em idosos. Em mulheres, relação entre menopausa, uso de estrogênio e ocorrência do ciclo osteoporose-fratura-dor parece ainda pouco esclarecida. Também a associação entre dor lombar e dor nos membros inferiores pode sugerir causas mecânicas ou outras causas, visto que osteoartrite e distúrbios circulatórios, comuns em idosas, nem sempre são fontes de dor.

Por fim, o uso de expressões como "outras causas mecânicas", "outras condições patológicas", "dor persistente pode representar a progressão e acumulação de problemas dolorosos segmentares", "parte de um problema doloroso mais generalizado" evidenciam ainda mais o cenário de indefinição encontrado na literatura utilizada para o estudo. Isso porque o uso dessas expressões demonstra que outras possibilidades de causas, pouco esclarecidas e compreendidas, podem estar na origem da dor lombar e dor nos membros inferiores de idosas. É possível que com o avanço da ciência e da tecnologia possam surgiu novos estudos que esclareçam, com maior grau de precisão, as questões que permanecem indefinidas.

\section{Referências}

1. World Health Organization - WHO. Ageing [cited 2008 Oct. 18]. Available from: http://www.who.int/topics/ ageing/en/index.html

2. World Health Organization - WHO. 10 facts on ageing and the life course [cited 2008 Oct. 18]. Available from: http://www.who.int/features/factfiles/ageing/en/

3. Instituto Brasileiro de Geografia e Estatística - IBGE. Síntese de indicadores sociais: uma análise das condições de vida da população brasileira 2007 [citado em 2008 out. 18]. Disponível em http://www.ibge.gov.br/home/ estatistica/populacao/condicaodevida/indicadoresminimos/sinteseindicsociais2007/indic_sociais2007.pdf

4. Fraiman AP. Coisas da idade. São Paulo: Hermes; 1988.

5. Mello TMT, Tourinho Filho H. Exercícios resistidos e o processo de envelhecimento: musculação na terceira idade? In: Pasqualotti A, Portella MR, Bettinelli LA. Envelhecimento humano: desafios e perspectivas. Passo Fundo: Ed da UPF; 2004. p. 194-210.

6. Guedes JM, Silveira RCR. Análise da capacidade funcional da população geriátrica institucionalizada na cidade de Passo Fundo-RS. RBCEH [periódico online] 2004 [citado em 17 out. 2008];1(2):10-21. Disponível em: http:// www.upf.br/seer/index.php/rbceh/article/viewfile/10/32.

7. Rudy TE, Weiner DK, Lieber SJ, Slaboda J, Boston JR. The impact of chronic low back pain on older adults: a comparative study of patients and controls. Pain [serial on the online] 2007 [cited 2008 Oct. 18];131(3):293-301. Available from: http://www.pubmedcentral.nih.gov/picrender.fcgi?artid=2065872\&blobtype=pdf

8. Leveille SG, Guralnik JM, Hochberg M, Hirsch R, Ferrucci L, Langlois J, et al. Low back pain and disability in older women: independent association with difficulty but not inability to perform daily activities. J Gerontol A Biol Sci Med Sci [serial on the online] 1999 [cited 2008 Oct. 16];54(10):487-93. Available from: http:/ /www.ncbi. nlm.nih.gov/pubmed/10568530?ordinalpos=7\&itool=EntrezSystem2.PEntrez.Pubmed.Pubmed_ResultsPanel. Pubmed_DefaultReportPanel.Pubmed_RVDocSum

9. Dellaroza MS, Pimenta CAM, Matsuo T. Prevalência e caracterização da dor crônica em idosos não institucionalizados. Cad. Saúde Pública [periódico online] 2007 [citado 17 out. 2008];23(5):1151-60. Disponível em: http:// www.scielo.br/pdf/csp/v23n5/17.pdf

10. Leveille SG, Bean J, Ngo L, McMullen W, Guralnik JM. The pathway from musculoskeletal pain to mobility difficulty in older disabled women. Pain [serial on the online] 2007 [cited 2008 Oct. 17];128(1-2):69-77. Available from: http:/ / www.pubmedcentral.nih.gov/articlerender.fcgi?tool=pubmed\&pubmedid=17055167 
11. Vogt MT, Lauerman WC, Chirumbole M, Kuller LH. A community-based study of postmenopausal white women with back and leg pain: health status and limitations in physical activity. J Gerontol A Biol Sci Med Sci [serial on the online] 2002 [cited 2009 Feb.12];57(8):544-50. Available from: http://www.ncbi.nlm.nih.gov/pubmed/121 45370?ordinalpos=1\&itool=EntrezSystem2.PEntrez.Pubmed.Pubmed_ResultsPanel.Pubmed_DiscoveryPanel. Pubmed_Discovery_RA\&linkpos $=2 \& \log \$=$ relatedarticles\&logdbfrom $=$ pubmed

12. Manchikanti L, Manchikanti KN, Cash KA, Singh V, Giordano J. Age-Related prevalence of facet-joint involvement in chronic neck and low back pain. Pain Physician [serial on the online] 2008 [cited 2009 Feb. 12];11(1):6775. Available from: http://www.painphysicianjournal.com/linkout_vw.php?issn=1533-3159\&vol=11\&page=67

13. Manchikanti L, Boswell MV, Singh V, Pampati V, Damron KS, Beyer CD. Prevalence of facet joint pain in chronic spinal pain of cervical, thoracic, and lumbar regions. BMC Musculoskelet Disord [serial on the online] 2004 [cited 2009 Feb. 12];5:15. Available from:http:/ / www.pubmedcentral.nih.gov/articlerender.fcgi?artid=441387\&tool=pmcentrez

14. Cecchi F, Debolini P, Lova RM, Macchi C, Bandinelli S, Bartali B, et al. Epidemiology of back pain in a representative cohort of Italian persons 65 years of age and older: the InCHIANTI study. Spine [serial on the online] 2006 [cited 2009Feb.13];31(10):1149-55. Available from: http://www.ncbi.nlm.nih.gov/pubmed/16648752?ordinalpos=1\&it ool=EntrezSystem2.PEntrez.Pubmed.Pubmed_ResultsPanel.Pubmed_DiscoveryPanel.Pubmed_Discovery_RA\& linkpos $=1 \& \log \$=$ relatedarticles\&logdbfrom $=$ pubmed

15. Lazaro L 4th, Quinet RJ. Low back pain: how to make the diagnosis in the older patient. Geriatrics [serial on the online] 1994 [cited 2009 Feb.13];49(9):48-53. Available from: http://www.ncbi.nlm.nih.gov/pubmed/808 8559?ordinalpos=1\&itool=EntrezSystem2.PEntrez.Pubmed.Pubmed_ResultsPanel.Pubmed_DiscoveryPanel. Pubmed_Discovery_RA\&linkpos $=2 \& \log \$=$ relatedreviews\&logdbfrom $=$ pubmed

16. Mazanec DJ. Evaluating back pain in older patients. Cleve Clin J Med [serial on the online] 1999 [cited 2009 Feb.13];66(2):89-91. Available from: http://www.ncbi.nlm.nih.gov/pubmed/9988954?ordinalpos=1\&itool=En trezSystem2.PEntrez.Pubmed.Pubmed_ResultsPanel.Pubmed_DiscoveryPanel.Pubmed_Discovery_RA\&linkpo $\mathrm{s}=5 \& \log \$=$ relatedreviews\&logdbfrom $=$ pubmed

17. BaumgartnerH.Lumbar pain in old age. Praxis (Bern 1994) [serial on the online] 1996 [cited 2009 Feb. 13];85(43):134753.Disponível em: http://www.ncbi.nlm.nih.gov/pubmed/8966436?ordinalpos=14\&itool=EntrezSystem2.PEntrez. Pubmed.Pubmed_ResultsPanel.Pubmed_DefaultReportPanel.Pubmed_RVDocSum

18. Wing PC. Rheumatology: 13. Minimizing disability in patients with low-back pain. CMAJ [serial on the online] 2001 [cited 2009 Feb. 13];164(10):1459-68. Available from: http://www.pubmedcentral.nih.gov/articlerender. fcgi?artid $=81075 \&$ tool $=$ pmcentrez

19. Henschke N, Maher CG, Refshauge KM. Screening for malignancy in low back pain patients: a systematic review. Eur Spine J [serial on the online] 2007 [cited 2009 Feb.13];16(10):1673-9. Available from: http:/ / www.pubmedcentral. nih.gov/articlerender.fcgi?tool=pubmed\&pubmedid=17566791

20. Burton RR. Parkinson's disease without tremor masquerading as mechanical back pain; a case report. JCCA J Can Chiropr Assoc [serial on the online] 2008 [cited 2009 Feb. 13]; 52(3):185-92. Available from: http://www. pubmedcentral.nih.gov/articlerender.fcgi? $\operatorname{artid}=2528272 \&$ tool $=$ pmcentrez

21. Ponka D, Kirlew M. Top 10 differential diagnoses in family medicine: low back pain. Can Fam Physician [serial on the online] 2007 [cited 2009 Feb. 13]; 53(6):1058. Available from: http:/ /www.pubmedcentral.nih.gov/articlerender. fcgi?tool=pubmed\&pubmedid $=17872787$

22. Eubanks JD, Lee MJ, Cassinelli E, Ahn NU. Prevalence of lumbar facet arthrosis and its relationship to age, sex, and race: an anatomic study of cadaveric specimens. Spine [serial on the online] 2007 [cited 2009 Feb. 13];32(19):2058-62. Available from: http://www.ncbi.nlm.nih.gov/pubmed/17762805?ordinalpos=1\&itool=EntrezSystem2.PEntrez. Pubmed.Pubmed_ResultsPanel.Pubmed_DiscoveryPanel.Pubmed_Discovery_RA\&linkpos $=5 \& \log \$=$ relatedarti cles\&logdbfrom=pubmed 
23. Borenstein D. Does osteoarthritis of the lumbar spine cause chronic low back pain? Curr Pain Headache Rep [serial on the online] 2004 [cited 2009 Feb. 13];8(6):512-7. Available from: http://www.ncbi.nlm.nih.gov/pubmed/155 09467?ordinalpos=1\&itool=EntrezSystem2.PEntrez.Pubmed.Pubmed_ResultsPanel.Pubmed_DiscoveryPanel. Pubmed_Discovery_RA\&linkpos $=5 \& \log \$=$ relatedreviews\&logdbfrom $=$ pubmed

24. Eubanks JD, Lee MJ, Cassinelli E, Ahn NU. Does lumbar facet arthrosis precede disc degeneration? A postmortem study. Clin Orthop Relat Res [serial on the online] 2007 [cited 2009 Feb. 13];464:184-9. Available from: http://www.ncbi.nlm.nih.gov/pubmed/17767079?ordinalpos=1\&itool=EntrezSystem2.PEntrez.Pubmed. Pubmed_ResultsPanel.Pubmed_DiscoveryPanel.Pubmed_Discovery_RA\&linkpos $=3 \& \log \$=$ relatedarticles\&log dbfrom=pubmed

25. Astin M, Lawton C, Hirst M. The prevalence of pain in a disabled population. Social Science \& Medicine [serial on the online] 1996 [cited 2009 Feb. 13];42(11):1457-64. Available from: http://www.sciencedirect. $\mathrm{com} /$ science?_ob=ArticleURL\&_udi=B6VBF-3VVVN75-R\&_user=10\&_rdoc $=1 \&$ ffmt $=$ \&_orig $=$ search\&_ sort $=\mathrm{d} \&$ view $=$ c\&_acct $=$ C000050221\&_version $=1 \& \_u r l V e r s i o n=0 \& \_u s e r i d=10 \& m d 5=350 f 577 \mathrm{~d} 47829031679$ b497a50735727

26. Leveille SG, Zhang Y, McMullen W, Kelly-Hayes M, Felson DT. Sex differences in musculoskeletal pain in older adults. Pain [serial on the online] 2005 [cited 2009 Feb. 13]; 116(3):332-8. Available from: http:/ /www.pubmedcentral. nih.gov/articlerender.fcgi?tool=pubmed\&pubmedid $=15982814$

27. Melton LJ 3rd. Epidemiology of spinal osteoporosis. Spine [serial on the online] 1997 [cited 2009 Feb. 15];22(24 Suppl):2s-11s. Available from: http://www.ncbi.nlm.nih.gov/pubmed/9431638?ordinalpos=1\&itool=EntrezSys tem2.PEntrez.Pubmed.Pubmed_ResultsPanel.Pubmed_DiscoveryPanel.Pubmed_Discovery_RA\&linkpos=5\&lo $\mathrm{g} \$=$ relatedreviews\&logdbfrom $=$ pubmed

28. Nevitt MC, Cummings SR, Stone KL, Palermo L, Black DM, Bauer DC, et al. Risk factors for a firstincident radiographic vertebral fracture in women $>$ or $=65$ years of age: the study of osteoporotic fractures. J Bone Miner Res [serial on the online] 2005 [cited 2009 Feb. 15];20(1):131-40. Available from: http://www.ncbi.nlm.nih.gov/pubmed/15619679?ordinalpos=1\&itool=EntrezSystem2.PEntrez.Pubmed. Pubmed_ResultsPanel.Pubmed_DiscoveryPanel.Pubmed_Discovery_RA\&linkpos $=2 \& \log \$=$ relatedarticle s\&logdbfrom $=$ pubmed

29. Hartvigsen J, Christensen K, Frederiksen H, Petersen HC. Genetic and environmental contributions to back pain in old age: a study of 2,108 danish twins aged 70 and older. Spine [serial on the online] 2004 [cited 2009 Feb. 15];29(8):897-901. Available from: http://www.ncbi.nlm.nih.gov/pubmed/15082992?ord inalpos=26\&itool=EntrezSystem2.PEntrez.Pubmed.Pubmed_ResultsPanel.Pubmed_DefaultReportPanel. Pubmed_RVDocSum

30. Nevitt MC, Ettinger B, Black DM, Stone K, Jamal SA, Ensrud K, et al. The association of radiographically detected vertebral fractures with back pain and function: a prospective study. Ann Intern Med [serial on the online] 1998 [cited 2009 Feb. 15];128(10):793-800. Available from: http://www.ncbi.nlm.nih.gov/pubmed/9599190?ordinalpos=1\&it ool=EntrezSystem2.PEntrez.Pubmed.Pubmed_ResultsPanel.Pubmed_DiscoveryPanel.Pubmed_Discovery_RA\& linkpos $=3 \& \log \$=$ relatedarticles\&logdbfrom $=$ pubmed

31. Krieg MA, Lamy O. Vertebral fracture: a major risk factor for osteoporosis. Praxis (Bern 1994) [serial on the online] 2004 [cited 2009 Feb. 15];93(9):321-8. Available from: http://www.ncbi.nlm.nih.gov/pubmed/15060 973?ordinalpos=1\&itool=EntrezSystem2.PEntrez.Pubmed.Pubmed_ResultsPanel.Pubmed_DiscoveryPanel. Pubmed_Discovery_RA\&linkpos $=5 \& \log \$=$ relatedreviews\&logdbfrom $=$ pubmed

32. Musgrave DS, Vogt MT, Nevitt MC, Cauley JA. Back problems among postmenopausal women taking estrogen replacement therapy: the study of osteoporotic fractures. Spine [serial on the online] 2001 [cited 2009 Feb. 15];26(14):1606-12. Available from: http://www.ncbi.nlm.nih.gov/pubmed/11462095?ordinalpos=7\&itool=En trezSystem2.PEntrez.Pubmed.Pubmed_ResultsPanel.Pubmed_DefaultReportPanel.Pubmed_RVDocSum 
33. Jones G, White C, Nguyen T, Sambrook PN, Kelly PJ, Eisman JA. Prevalent vertebral deformities: relationship to bone mineral density and spinal osteophytosis in elderly men and women. Osteoporos Int. [serial on the online] 1996 [cited 2009 Feb. 15];6(3):233-9. Available from: http://www.ncbi.nlm.nih.gov/pubmed/8783298?ordinalpos=1\&it ool=EntrezSystem2.PEntrez.Pubmed.Pubmed_ResultsPanel.Pubmed_DiscoveryPanel.Pubmed_Discovery_RA\& linkpos $=2 \& \log \$=$ relatedarticles\&logdbfrom $=$ pubmed

34. Edmond SL, Kiel DP, Samelson EJ, Kelly-Hayes M, Felson DT. Vertebral deformity, back symptoms, and functional limitations among older women: the Framingham Study. Osteoporos Int. [serial on the online] 2005 [cited 2009 Feb. 15];16(9):1086-95. Available from: http://www.ncbi.nlm.nih.gov/pubmed/15726295?ordinalpos=1\&it ool=EntrezSystem2.PEntrez.Pubmed.Pubmed_ResultsPanel.Pubmed_DiscoveryPanel.Pubmed_Discovery_RA\& linkpos $=1 \& \log \$=$ relatedarticles\&logdbfrom $=$ pubmed

35. Ettinger B, Black DM, Nevitt MC, Rundle AC, Cauley JA, Cummings SR, et al. Contribution of vertebral deformities to chronic back pain and disability. The Study of Osteoporotic Fractures Research Group. [serial on the online] 1992 [cited 2009 Feb. 15];7(4):449-56. Available from: http://www.ncbi.nlm.nih.gov/pubmed/153517 2?ordinalpos=6\&itool=EntrezSystem2.PEntrez.Pubmed.Pubmed_ResultsPanel.Pubmed_DefaultReportPanel. Pubmed_RVDocSum

36. Vogt MT, Nevitt MC, Cauley JA. Spine. Back problems and atherosclerosis. The Study of Osteoporotic Fractures [serial on the online] 1997 [cited 2009 Feb.15];22(23):2741-7. Available from: http:/ /www.ncbi.nlm.nih.gov/pubmed /9431608?ordinalpos=1\&itool=EntrezSystem2.PEntrez.Pubmed.Pubmed_ResultsPanel.Pubmed_DiscoveryPanel. Pubmed_Discovery_RA\&linkpos $=1 \& \log \$=$ relatedarticles\&logdbfrom $=$ pubmed

37. Pahor M, Guralnik JM, Wan JY, Ferrucci L, Penninx BW, Lyles A, et al. Lower body osteoarticular pain and dose of analgesic medications in older disabled women: the Women's Health and Aging Study. Am J Public Health [serial on the online] 1999 [cited 2009 Feb. 13]; 89(6):930-4. Available from: http://www.pubmedcentral.nih.gov/ pagerender.fcgi?artid $=1508667 \&$ tool $=$ pmcentrez\&pageindex $=1$

38. Creighton D, Krauss J, Kondratek M, Huijbregts PA, Will A. Use of anterior tibial translation in the management of patellofemoral pain syndrome in older patients: a case series. J Man Manip Ther [serial on the online] 2007 [cited 2009 Feb. 13];15(4):216-24. Available from: http://www.pubmedcentral.nih.gov/articlerender.fcgi?artid=2 565629\&tool=pmcentrez

39. Cecchi F, Mannoni A, Molino-Lova R, Ceppatelli S, Benvenuti E, Bandinelli S, et al. Epidemiology of hip and knee pain in a community based sample of Italian persons aged 65 and older. Osteoarthritis Cartilage [serial on the online] 2008 [cited 2009 Feb. 14];16(9):1039-46. Available from: http:/ /www.ncbi.nlm.nih.gov/pubmed/1834 3164?ordinalpos=3\&itool=EntrezSystem2.PEntrez.Pubmed.Pubmed_ResultsPanel.Pubmed_DefaultReportPanel. Pubmed_RVDocSum

40. Leveille SG, Guralnik JM, Ferrucci L, Hirsch R, Simonsick E, Hochberg MC. Foot pain and disability in older women. Am J Epidemiol [serial on the online] 1998 [cited 2009 Feb. 14];148(7):657-65. Available from: http:// www.ncbi.nlm.nih.gov/pubmed/9778172

Recebido: 28/04/2009

Received: 04/28/2009

Aprovado: $12 / 05 / 2010$

Approved: 05/12/2010 